\title{
A Polar Patrol Balloon Observation of Cosmic-Ray Protons and Auroral X-Rays in Antarctica
}

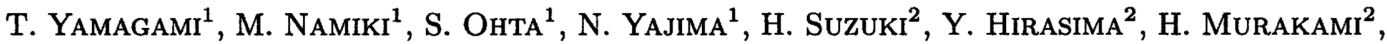 \\ K. Morimoto ${ }^{2}$, I. YAMAGIWA ${ }^{2 *}$, M. NAKAGAWA ${ }^{3}$, T. TAKAHASHI ${ }^{3}$, S. MURAKAMI ${ }^{3}$, J. NishimURA ${ }^{4}$, \\ Y. TONEGAWA ${ }^{5}$, M. EJIRI ${ }^{6}$, N. SATO ${ }^{6}$, T. KOHNO ${ }^{7}$, and M. KODAMA \\ ${ }^{1}$ The Institute of Space and Astronautical Science, Sagamihara, Kanagawa 229, Japan \\ ${ }^{2}$ Faculty of Science, Rikkyo University, Nishi-Ikebukuro, Toshima-ku, Tokyo 171, Japan \\ ${ }^{3}$ Faculty of Science, Osaka City University, Sumiyoshi-ku, Osaka 558, Japan \\ ${ }^{4}$ Faculty of Engineering, Kanagawa University, Rokkakubashi, Kanagawa-ku, Yokohama 221, Japan \\ ${ }^{5}$ Faculty of Science, Tokai University, Hiratsuka, Kanagawa 259-12, Japan \\ ${ }^{6}$ National Institute of Polar Research, Kaga, Itabashi-ku, Tokyo 173, Japan \\ ${ }^{7}$ Institute of Physical and Chemical Research, Wako, Saitama 351-01, Japan
}

(Received May 31, 1994; Revised September 2, 1994; Accepted September 2, 1994)

\begin{abstract}
A Polar Patrol Balloon observation of cosmic ray protons $(E \geq 100 \mathrm{MeV})$ was carried out in Antarctica, also energetic X-ray $(E=30-120 \mathrm{keV})$ observations of auroral, solar and/or cosmic origins as well. The Polar Patrol Balloon No. 6 (PPB\#6) was launched from Syowa Station on January 5, 1993 and drifted westward by 1.5 circumpolar rounds over Antarctica, covering $6-13 \mathrm{~g} / \mathrm{cm}^{2}$ atmospheric depth and $63^{\circ} \mathrm{S}-70^{\circ} \mathrm{S}$ geographic latitude. A total of 584 hours of observations was obtained. During the period, data telemetry and balloon tracking were performed by a multi-ID ARGOS system, through which the all data were acquired every 30 seconds. A preliminary report of fluxes and spatial distributions of cosmic ray protons and auroral X-rays is presented. Some features of the results are summarized as follows. 1) Proton fluxes measured in the polar cusp region still reveal a progressive increase at higher latitudes above the cosmic-ray latitude knee. 2) The differential energy spectrum of cosmic ray protons measured near the solar minimum is consistent with observations in 1967 and 1977. 3) Distinct enhancements of auroral X-rays were often observed, particularly dominant in the magnetic local dayside time.
\end{abstract}

\section{Introduction}

Recently, several advantages of balloon experiments in Antarctica have become attractive, including high feasibility of a very long-lived flight due to a little noon-midnight temperature difference in austral summer. Long duration flight was first demonstrated by using a superpressure balloon (e.g., Levanon et al., 1977). But such balloons cannot carry heavier payloads to altitudes above about $30 \mathrm{~km}$, because the technical development for large-scale superpressure balloons essential for such extended flights is not so easy.

The Polar Patrol Balloon (PPB) project, planned by National Institute of Polar Research and the Institute of Space and Astronautical Science, started in 1987, to establish long-duration capability of zero-pressure balloons above $30 \mathrm{~km}$ height in Antarctica (Nishimura et al., 1985; Hirasawa et al., 1990). Up to October 1991, five PPBs including two test flights had been launched from Syowa Station $\left(69.0^{\circ} \mathrm{S}, 39.6^{\circ} \mathrm{E}\right)$. Two of them succeeded to drift almost one circumpolar path during a few weeks, counterclockwise in austral summer and clockwise in spring (Kanzawa, 1992; Ejiri et al., 1993).

${ }^{*}$ Now at Faculty of Science, Saitama University, Urawa, Saitama 338, Japan. 
The 34th Japanese Antarctic Research Expedition members performed three PPB flights in December 1992-January 1993. The final and largest one $\left(59,467 \mathrm{~m}^{3}\right.$ in volume) launched on January 5, 1993, continued to fly until the end of January. With this flight (hereinafter referred to as PPB\#6) 1.5 circumpolar navigation was accomplished for the first time. The resulting total observation time of flight was 584 hours at above $30 \mathrm{~km}$ height and above $49^{\circ}$ invariant latitude. This was good enough to measure cosmic-ray proton and helium fluxes with a fairly good statistics, and to monitor auroral $\mathrm{X}$-rays over vast spatial extents.

The PPB\#6 balloon experiment was performed during the current recovery to the solar minimum in order to search the following three objects: (1) temporal and spectral characteristics of cosmic and/or solar protons, (2) energy spectra of cosmic ray isotopes, $\mathrm{p}, \mathrm{d}, \mathrm{t}$, helium and elemental abundance of CNO components, and (3) hard X-ray spectra of auroral, solar, and cosmic origins and their spatial distributions over the south polar region. Some of the results obtained from proton and X-ray measurements are described in this paper. This cosmic-ray experiment followed the first balloon observation of cosmic-ray proton and helium components in the vicinity of Syowa Station (Tamai et al., 1971).

\section{Flight Trajectory of PPB\#6}

PPB\#6 was launched from Syowa Station at 0855 UT on January 5, 1993, and drifted westward at a ceiling altitude between $30 \mathrm{~km}$ and $36 \mathrm{~km}$. As shown in Fig. 1, PPB\#6 circumnavigated over $580^{\circ}$ of longitude to January 31 , covering the geographic latitude range from $63^{\circ} \mathrm{S}$ to $70^{\circ} \mathrm{S}$. It took about 16 days for one circumnavigation. This corresponds to $49^{\circ}-81^{\circ}$ invariant latitude survey, as seen in the middle panel of Fig. 2. Consequently, a spatial survey was accomplished over the wide region from the cusp to the low-latitude boundary of the auroral zone, which is favorable for observation of auroral X-rays and low energy cosmic-ray components.

\section{Instrumentation}

The following payload of observing equipment, house-keeping (HK) and telemetry systems was carried by PPB\#6.

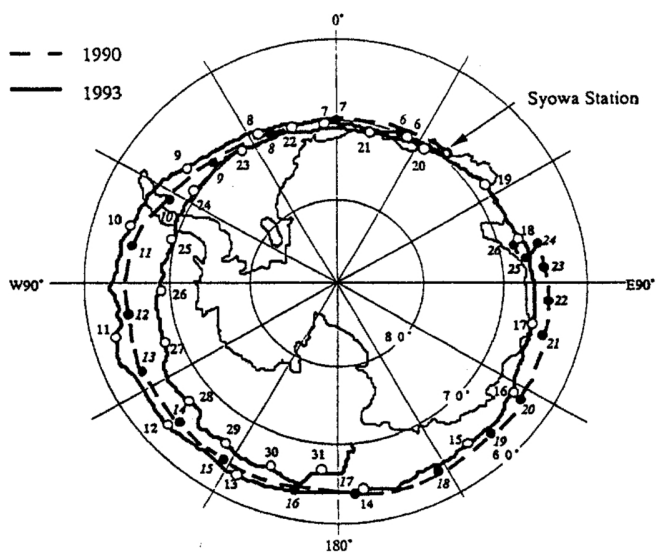

Fig. 1. Trajectory of Polar Patrol Balloon No. 6 (PPB\#6) launched from Syowa Station $\left(69.0^{\circ} \mathrm{S}, 39.6^{\circ} \mathrm{E}\right)$ on January 5,1993 , on the geographical coordinates is presented by a solid line. A broken line shows the trajectory of PPB\#2 launched on January 5, 1990. Numerical figures attached indicate the dates at $0 \mathrm{~h}$ UT. 


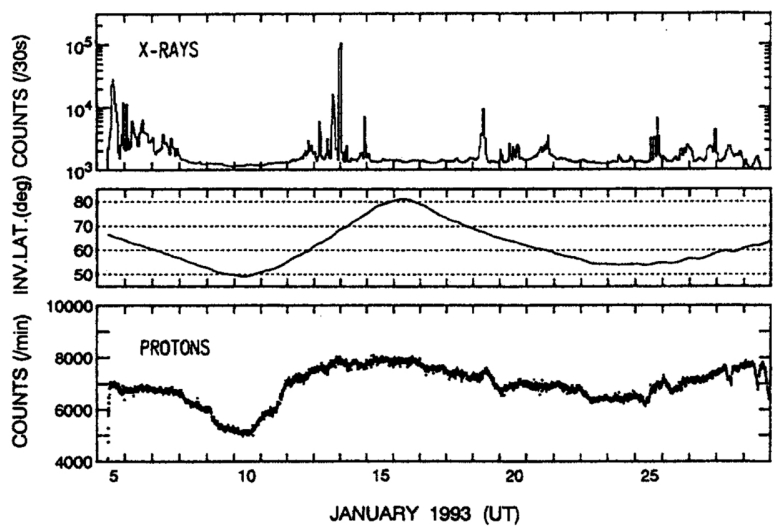

Fig. 2. Time profile of hourly averaged 30-120 keV X-ray counts (top), invariant latitudes through which PPB\#6 passed (middle) and 5-min averaged 100-150 MeV cosmic-ray protons (bottom). The proton data were corrected for the altitude dependence by converting to the counting rates at $10 \mathrm{~g} / \mathrm{cm}^{2}$.

\subsection{Observational instruments}

In order to measure the cosmic ray components and X-rays mentioned in Section 1, three detection systems (D1, D2 and D3) were used. D1: Proton measurements: a scintillation counter, using $2^{\prime \prime}$ dia. $\times 100 \mathrm{~mm}$ thick $\mathrm{CsI}(\mathrm{Tl})$ crystal (geometric factor $127.5 \mathrm{~cm}^{2} \mathrm{str}$ ). Spectrum measurements were made in five different energy bands; 100-150 MeV, $150-200 \mathrm{MeV}, 200-250 \mathrm{MeV}$, 250-300 MeV and $>300 \mathrm{MeV}$. This D1 detection system was calibrated with $80 \mathrm{MeV}$ and 135 $\mathrm{MeV}$ protons from the RIKEN ring cyclotron. Energy resolution was 6.5\% (FWHM). D2: Cosmic-ray isotopes and CNO measurements: a telescope consisting of 3 SSDs, each one, (28 $\mathrm{mm} \times 28 \mathrm{~mm}$ ) size $\times 0.5 \mathrm{~mm}$ thick, and a $\mathrm{CsI}(\mathrm{Tl}), 2^{\prime \prime}$ dia. $\times 100 \mathrm{~mm}$ thick (geometric factor 0.088 $\mathrm{cm}^{2} \mathrm{str}$ ). Proton energy channels are five by $50 \mathrm{MeV}$ steps between 100 and $350 \mathrm{MeV}$, and one above $350 \mathrm{MeV}$. D3: Hard X-ray measurements: a NaI(Tl) scintillation counter, $2^{\prime \prime}$ dia. $\times 5 \mathrm{~mm}$ thick, with a full view-field of $165^{\circ}$ (geometric factor $52.7 \mathrm{~cm}^{2} \mathrm{str}$ ) and energy range is 30-120 $\mathrm{keV}$.

\subsection{Auto-ballast system}

The flight altitude of PPB\#6 was automatically controlled by an auto-ballast system with a high-sensitivity pressure gauge. The altitude varied periodically with a small diurnal wave of a few $\mathrm{g} / \mathrm{cm}^{2}$ amplitude, until the final stage of flight after January 28 when the auto-ballast system operation was exhausted. This experience validates the use of such an auto-ballast control system. Averaged period of the diurnal wave was 25.7 hours.

\subsection{Data acquisition and telemetry system}

Data transmission for PPB\#6 was provided by the multiple-identify (multi-ID) ARGOS system that contains 40 ID channels. Because of the small transmission rate of the ARGOS system, $150 \mathrm{k}$ bytes/hour, a total of forty sets of data including the HK-data were transmitted after $30 \mathrm{sec}$ sampling. One ID channel consists of 32 words one byte long. The underlying premise of successful data was that PPB\#6 had a chance of encounter at least once an hour with the ARGOS/NOAA satellites. As a result, the encounter was not regular but arbitrary, so that about $20 \%$ of the total observation data were missed. 


\section{Observational Results}

Brief notes on the observational results obtained by PPB\#6 are described in the following subparagraphs 4.1-4.2. Details of the studies referred to these will be given in separate papers, including cosmic-ray heavier nuclei.

\subsection{Auroral $X$-rays}

Figure 2 shows the time profiles of 30-120 keV X-rays during the entire flight (top) and of the invariant latitudes through which PPB\#6 passed (middle). As seen from a comparison between the two panels of Fig. 2, distinct enhancements of auroral X-rays were often observed in the vicinity of $60^{\circ}-70^{\circ}$ invariant latitude. During the period of the balloon flight, $K_{p}$ indices were moderate (between 0 and 6, average 3-). The spatial distribution of X-ray fluxes is shown on geomagnetic coordinates in Fig. 3. Magnetic local times (MLTs) and invariant latitudes were interpolated from location data of ARGOS system, using the IGRF model. A wide region of pronounced X-ray enhancement is present at the dayside. We note the total time intervals of X-ray enhancement with total counts of $>3000$ counts/30 sec (about two times background). Seventy-nine percent and $78 \%$ of the total time were found in $60^{\circ}-70^{\circ}$ invariant latitudes, $6-18$ $\mathrm{h}$ MLT sector, respectively. It is therefore likely that dayside X-ray aurorae were predominant throughout the PPB\#6 flight.

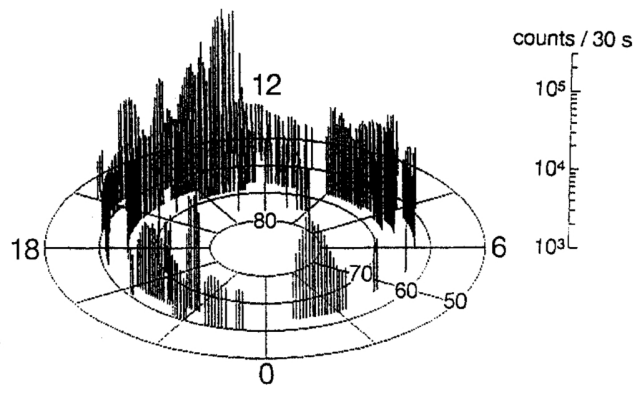

Fig. 3. Spatial distribution of X-ray counting rates, shown by vertical lines whose lengths represent counting rates given in the legend, on the polar coordinates of MLT and invariant latitude. MLTs and invariant latitudes in $\mathrm{X}$-ray events were calculated from location data supplied by ARGOS system.

\subsection{Cosmic-ray protons}

The time profile of proton counting rates $(E=100-150 \mathrm{MeV})$ obtained by the detector D1 is shown in the bottom panel of Fig. 2, converting to counting rates at $10 \mathrm{~g} / \mathrm{cm}^{2}$. It is evident from a comparison with the middle panel that the proton counts generally vary in response to invariant latitude. To see more clearly latitude dependence, proton fluxes within a limited narrow altitude $\left(7.8-8.2 \mathrm{~g} / \mathrm{cm}^{2}\right)$ were plotted against the invariant latitude so as to minimize the altitude dependence. As seen in Fig. 4, the proton fluxes obviously increase up to near $60^{\circ}$, and a smaller positive gradient is significant at latitudes higher than $60^{\circ}$. It is noticeable that proton fluxes still increase even at high latitudes above the cosmic-ray latitude knee around $60^{\circ}$. Figure 5 shows the differential energy spectra of protons measured by D2 in the vicinity of $55^{\circ}$ and $80^{\circ}$. The spectrum obtained at $55^{\circ}$ is consistent, within one sigma error, with that measured near $40^{\circ}$ (Gloeckler and Jokipii, 1967), whereas the energy spectrum yielded at $80^{\circ}$ is higher than 


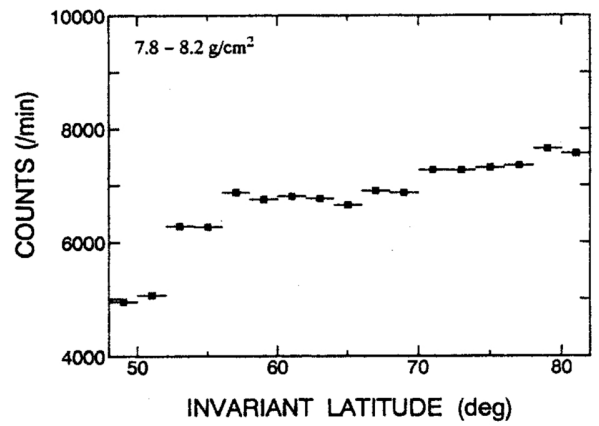

Fig. 4.

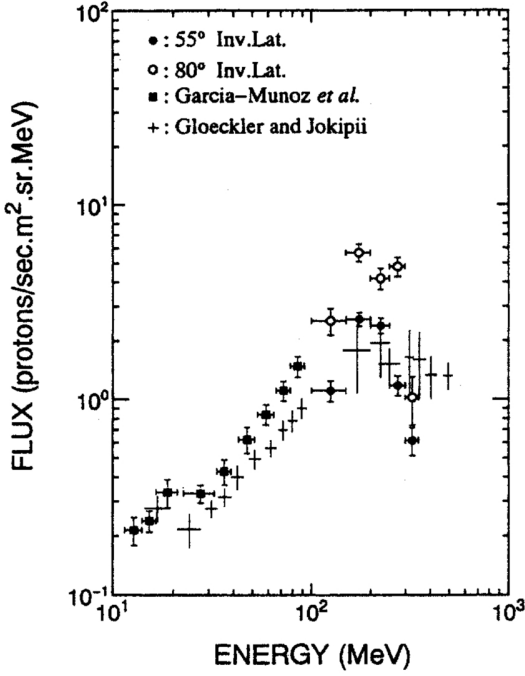

Fig. 5.

Fig. 4. Invariant latitude dependence of 100-150 MeV cosmic-ray proton fluxes measured by the D1 detector. The counting rates per one minute averaged during 5 minutes obtained at the narrow altitude range of 7.8-8.2 $\mathrm{g} / \mathrm{cm}^{2}$ are plotted so as to minimized the altitude dependence. Statistical errors of fluxes are negligibly small.

Fig. 5. Energy spectra of cosmic-ray protons measured at a depth of $6-8 \mathrm{~g} / \mathrm{cm}^{2}$; ours at $55^{\circ}$ (solid circles) and $80^{\circ}$ (open circles) invariant latitude; by satellite, Garcia-Munoz et al. (solid square), and Gloeckler and Jokipii (cross).

that of $55^{\circ}$ and comparable with the extrapolation of the spectrum measured by IMP-8 in space (Garcia-Munoz et al., 1977).

\section{Discussion}

The altitude variations of PPB\#6 were the day-to-day wave. The valley level, appearing once a day, was almost constant throughout the flight, while the peak altitude was subject to the long-term wave. The latter seems to be caused dominantly by the sunset influence. The period of the diurnal wave depends on the float velocity of PPB\#6, or the flight altitude, and was 25.7 hours on the average. This period was longer than 24 hours, because the balloon traveled westward. This information on flight technology will be useful for studies of thermal and dynamical behavior of the PPB.

We note that the PPB\#6 flight trajectory was very close to that of PPB\#2, launched on January 5, 1990. PPB\#2 trajectory is shown by a broken line of Fig. 1 (Kadokura et al., 1991). Although PPB\#2 took somewhat longer circumpolar period (roughly estimated to be 19 days from a total of the $3 / 4$ round flight) because of the lower ceiling altitude $(25-32 \mathrm{~km})$, the both flight cruises are well resemble as the whole. It is, therefore, likely that a PPB launched before and after the beginning of a new year will take a definite flight route resembling the Fig. 1 trajectories, though the circumpolar period will depend upon the flight altitude. Thus the PPB promises a long period and wider region observation possible.

We emphasize that the widespread distribution of auroral X-rays as shown in Fig. 2 comes from only one balloon flight carrying a single unique detector. This kind of broad mapping was previously possible only by satellite technique (e.g., Imhof $e t$ al., 1985), which is based on a number 
of straight fast crossing surveys over the polar regions with about one hour spacing between any one crossing and the next. Since the circumpolar survey by the PPB is generally conducted in quite a different way from these satellite paths, the spatial distribution thereby obtained will explore a new view-field of auroral study concerned with energetic particle precipitations.

The time profiles of proton fluxes shown in Fig. 2 shows a systematic variation that corresponds to that of the invariant latitude. In other words, the proton fluxes increase with higher latitude even in the cusp region. It is very important to study the spectral difference between the near cusp region and the higher cut-off rigidity area. At $55^{\circ}$ invariant latitude, the cosmic-ray proton spectrum observed by PPB\#6 is fairly consistent with the measurements compiled by Gloeckler and Jokipii (1967), and Garcia-Munoz et al. (1977) within an error of one sigma. However, the spectrum at $80^{\circ}$ invariant latitude is clearly shifted upward relative to that at $55^{\circ}$. The majority of particles measured at balloon altitude of $6-8 \mathrm{~g} / \mathrm{cm}^{2}$ is thought to be possibly albedo protons of atmospheric origin, except for a small fraction of directly entrant cosmic protons ranging from 100 to $500 \mathrm{MeV}$ (0.5-1.1 GV) (Gloeckler and Jokipii, 1967; Verma, 1967). Accordingly, the enhanced proton flux observed at the cusp region must be investigated further in connection with the re-entrant albedo, helium flux or other factors.

This balloon experiment was conducted by JARE-34 members under the sponsorship of National Institute of Polar Research and the Institute of Space and Astronautical Science. Thanks are due to the PPB Working Group members for their cooperative work throughout this campaign. One of the authors (M.N.) is thankful to Center for Planning and Information Systems, the Institute of Space and Astronautical Science for data processing. Onboard CPU systems and electronic systems of the detectors were fabricated by the Clear Pulse Company, and the ARGOS system was fabricated by the Toyocom Company.

\section{REFERENCES}

Ejiri, M., A. Kadokura, T. Hirasawa, N. Sato, R. Fujii, H. Miyaoka, J. Nishimura, N. Yajima, T. Yamagami, S. Kokubun, H. Fukunishi, M. Yamanaka, and M. Kodama, Polar patrol balloon experiment in Antarctica, Adv. Space Res., 13, 127-130, 1993.

Garcia-Munoz, M., G. M. Mason, and J. A. Simpson, The appearance of super fluxes of quiet time cosmic rays, 15th Inter. Conf. Cosmic Rays, Plovdiv, 3, 209-214, 1977.

Gloeckler, G. and J. R. Jokipii, Solar modulation and the energy density of galactic cosmic rays, Ap. J. Lett., 148, L41-L46, 1967.

Hirasawa, T., M. Ejiri, N. Sato, R. Fujii, H. Miyaoka, A. Kadokura, J. Nishimura, N. Yajima, T. Yamagami, S. Kokubun, H. Fukunishi, M. D. Yamanaka, and M. Kodama, Polar patrol balloon experiment during 1991-1993, Proc. ISTS Symp., 1990.

Imhof, W. L., H. D. Voss, D. W. Datlowe, and J. Mobilia, Bremsstrahlung X ray images of isolated electron patches at high latitude, J. Geophys. Res., 90, 6515-6524, 1985.

Kadokura, A., M. Ejiri, S. Ohta, and H. Akiyama, Polar Patrol Balloon (PPB) experiment of the 30th Japanese Antarctic Research Expedition (1989-1990), Antarctic Rec., 35, 43-154, 1991.

Kanzawa, H., Polar patrol balloon (PPB) launched for observation of the Antarctic ozone hole of 1991, SCAR Newslett., No. 2, 14-15, 1992.

Levanon, N., P. R. Julian, and V. E. Suomi, Antarctic topography from balloons, Nature, 268, 514-516, 1977.

Nishimura, J., M. Kodama, K. Tsuruda, H. Fukunishi, and Co-members of PPB Working Group, Feasibility studies of "Polar Patrol Balloon", Adv. Space Res., 5, 87-90, 1985.

Tamai, E., T. Sakai, N. Narita, K. Ogura, S. Kogami, M. Kusunose, and M. Kodama, Low energy cosmic ray protons, He, and neutral particles over Syowa Station, Antarctica, 12th Inter. Conf. Cosmic Rays, Hobart, 2, 555-560, 1971.

Verma, S. D., Measurement of the charged splash and re-entrant albedo of cosmic radiation, J. Geophys. Res., 72, 915-925, 1967. 